\title{
UNANTICIPATED ADMISSIONS AFTER SAME DAY SURGERY IN A TEACHING HOSPITAL
}

\author{
Etulain Santos M.J., Monteserín C., Soriano L, Olmedo J.,López Martinez M., Zaballos M. \\ General University Hospital Gregorio Marañón. Madrid, Spain
}

Background and Goal of Study: Improvements in surgical and anaesthetic techniques in day surgery have resulted in low mortality and morbidity. The unplanned admission on same day of surgery is considered to be an important measure of the quality of ambulatory surgical units.

Our surgery centre was accredited in 2013, and then we have updated our protocols of multimodal pain management and those of prevention of postoperative nausea and vomiting. The objective of our study was to evaluate the unanticipated hospital admission rate from our day surgery unit and to analyse the reasons for admission.

Materials and Methods: A review of all unanticipated hospital admission from 2013 to march 2016 was done. The variables evaluated were: demographics, American Society of Anaesthesiologists (ASA) classification, type of anaesthesia, time of surgery, surgical speciality and reason for admission.

\begin{tabular}{l|l}
\multicolumn{1}{|c|}{ GENERAL CHARACTERISTICS } & \\
\hline Age & $53 \pm 17$ \\
\hline Gender F/M n (\%) & $74(43) ; 76(44)$ \\
\hline Weight (kg) & $74 \pm 15$ \\
\hline Height (cm) & $167 \pm 9$ \\
\hline Surgical time (min) & $119 \pm 61$ \\
\hline Bed time URPA I (min) & $288 \pm 210$ \\
\hline Seat time (min) & $160 \pm 97$ \\
\hline ASA I/II/III/IV n (\%) & $48(28) ; 77(45) ; 24(14) ; 1(0,6)$ \\
\hline Mallampati I/II/III/IV n (\%) & $70(41) ; 47(27) ; 29(17) ; 4(2)$
\end{tabular}

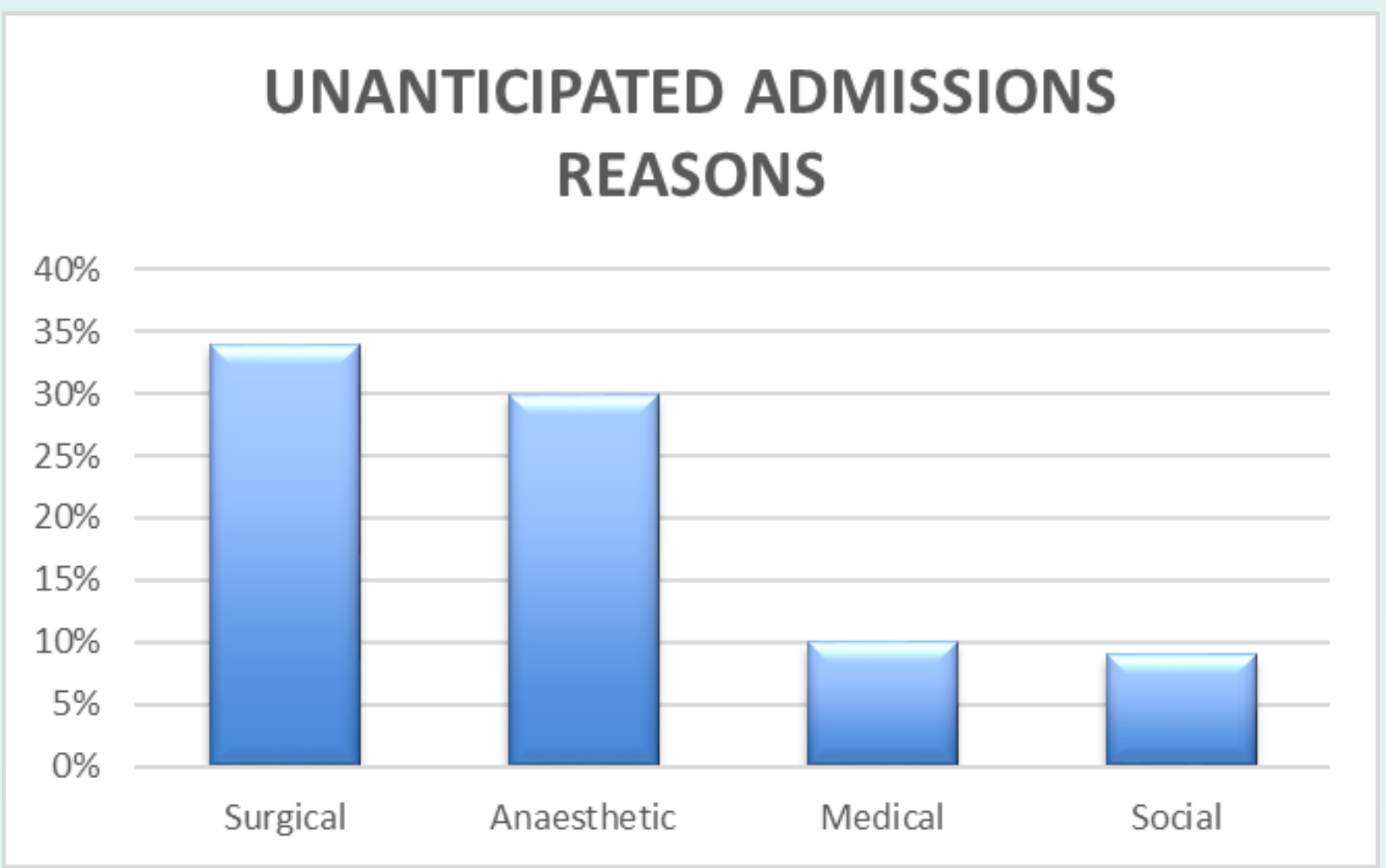

* Retrospective Evaluation of Unanticipated Admissions and Readmissions After Same Day Surgery and Associated Costs. Kim C. Coley. J. Clin. Anesth., vol. 14, August 2002

**Predictors of unanticipated admission following ambulatory surgery: a retrospective case-control study. Amanda Whippey, MD. Can J Anesth/J Can Anesth (2013) 60:675

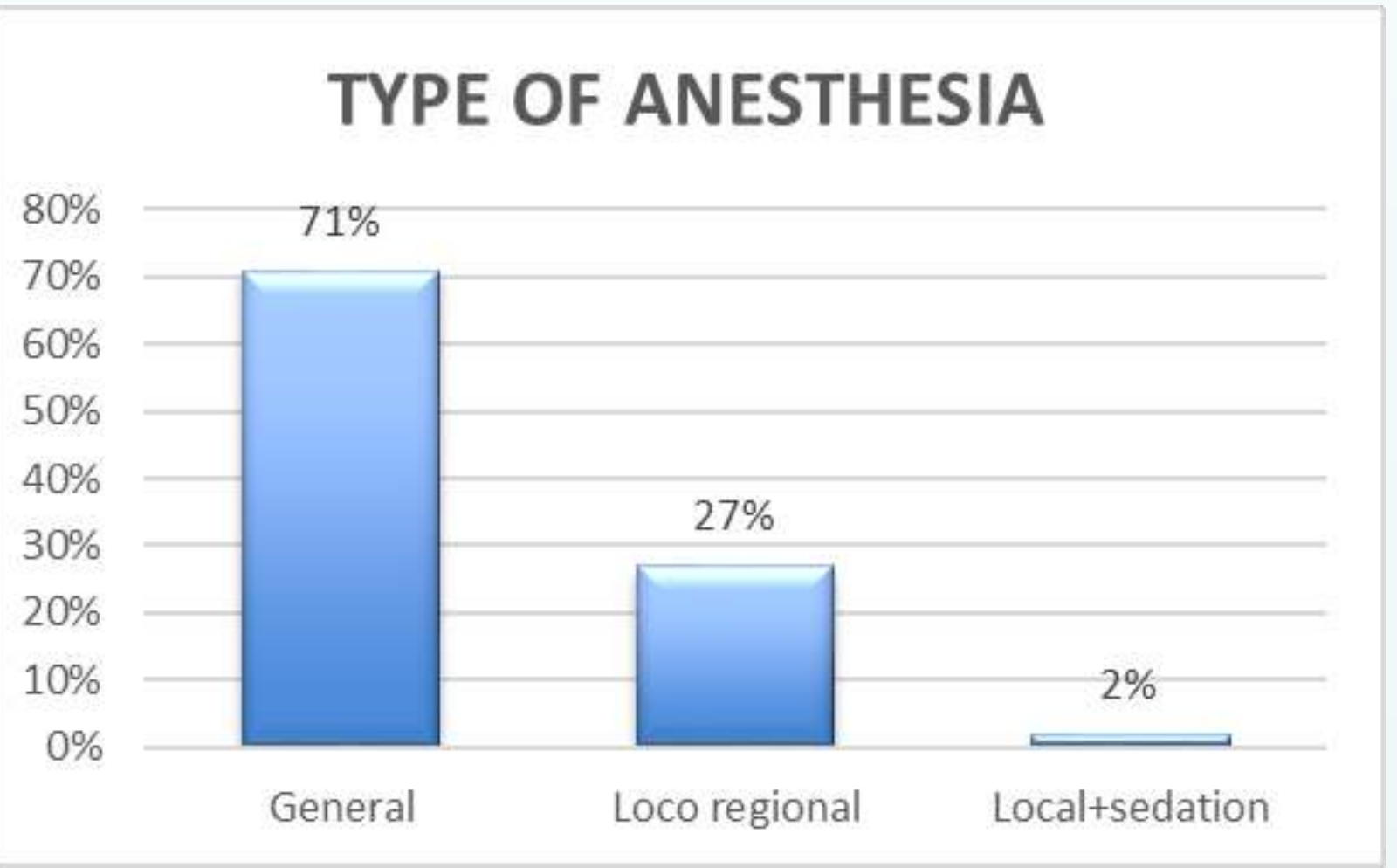

Results: A total of 8491 procedures were performed during the study period. The overall admission rate was $2 \%$. Most of the admissions were related to surgical (34\%) anaesthetic (30\%) and medical (10\%) reasons. Social reasons were present in $9 \%$ of cases. General (37\%) and ENT (26\%) surgeries were associated with and increasing risk of unanticipated admission. Pain (52\%) and postoperative nausea and vomiting (PONV) (32\%) were present as the principal anaesthetic related to admissions. The ASA status was not linked with unanticipated admission. Seventy percent of the admitted patients received general anaesthesia. Surgeries ending in the afternoon were not related with unanticipated admission (20\%).

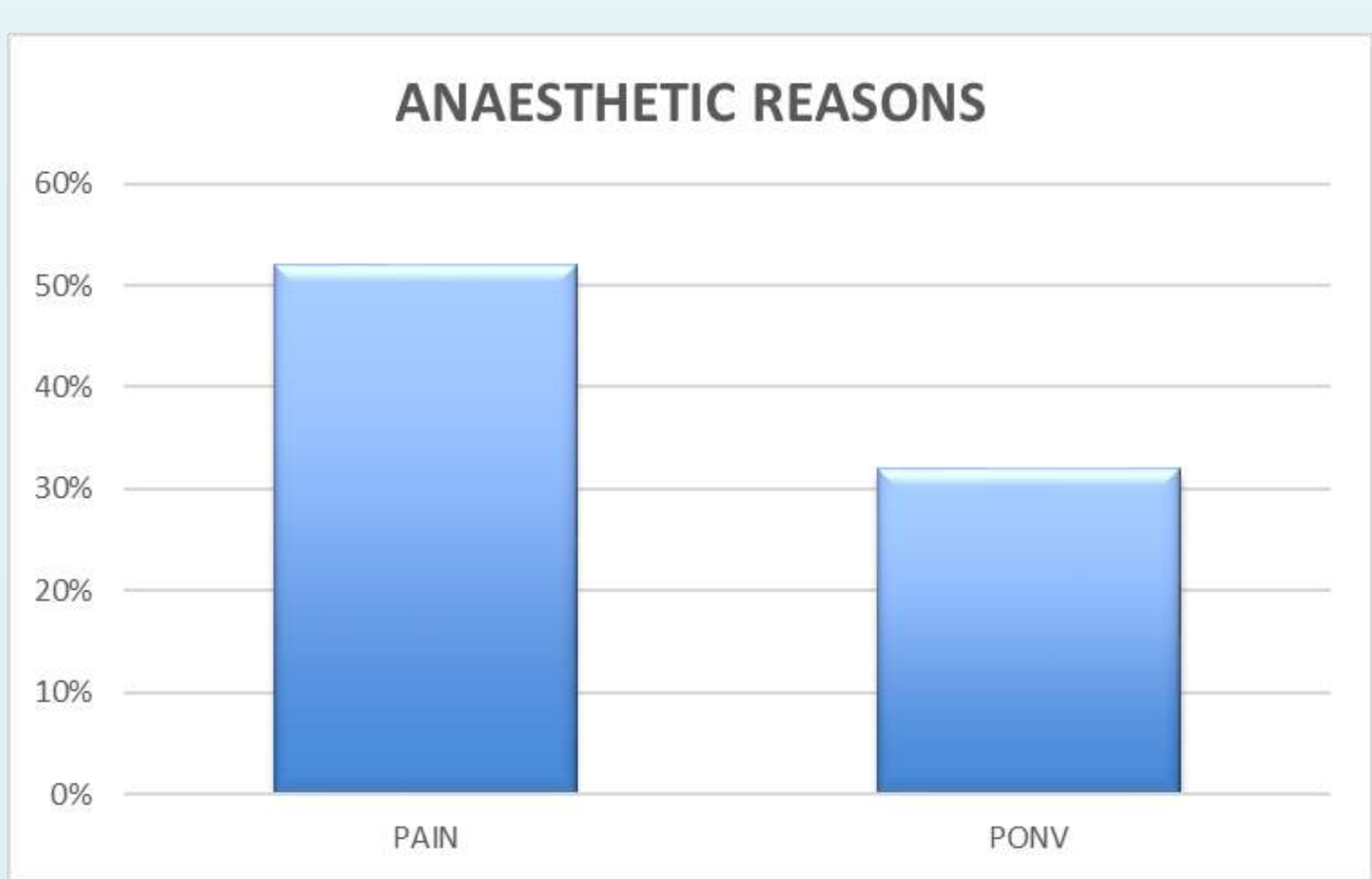

Conclusions: A large number of admissions were found to be preventable causes. Pain accounted for more than fifty percent of the anaesthetics linked admissions. Efforts to manage pain and PONV should be attempted in order to improve the efficiency of the ambulatory surgical centres. Nevertheless a contribution from unexpected scenarios is unavoidable. 